\title{
On Dispersion Relation for Gravity Waves in the Presence of Condensate.
}

\author{
A. O. Korotkevich \\ Department of Mathematics and Statistics, University of New Mexico, MSC01 1115, 1 University of New \\ Mexico, Albuquerque, NM 87131-0001, USA \\ L. D. Landau Institute for Theoretical Physics RAS, 2 Kosygin Str., Moscow, 119334, Russian Federation
}

\begin{abstract}
During direct numerical simulation of isotropic turbulence of surface gravity waves in the framework of Hamiltonian equations the formation of long wave condensate was observed. Here we study the influence of the condensate presence on the dispersion relation for the gravity waves. Formation of the two side bands, corresponding to the interaction with condensate, was observed. Distortion of the dispersion relation can be a factor influencing the spectra of inverse and direct turbulent cascades.
\end{abstract}

Keywords: gravity waves, inverse cascade, dispersion

PACS: 47.27.ek, 47.35.-i, 47.35.Jk

\section{INTRODUCTION.}

Here and further we shall follow notations from [1]. We consider a potential flow of ideal incompressible fluid. System is described in terms of weakly nonlinear equations $[2,3]$ for surface elevation $\eta(\vec{r}, t)$ and velocity potential at the surface $\psi(\vec{r}, t)(\vec{r}=\overrightarrow{(x, y)})$

$$
\begin{array}{r}
\dot{\eta}=\hat{k} \psi-(\nabla(\eta \nabla \psi))-\hat{k}[\eta \hat{k} \psi]+\hat{k}(\eta \hat{k}[\eta \hat{k} \psi])+\frac{1}{2} \Delta\left[\eta^{2} \hat{k} \psi\right]+\frac{1}{2} \hat{k}\left[\eta^{2} \Delta \psi\right]+\widehat{F}^{-1}\left[\gamma_{k} \eta_{k}\right], \\
\dot{\psi}=-g \eta-\frac{1}{2}\left[(\nabla \psi)^{2}-(\hat{k} \psi)^{2}\right]-[\hat{k} \psi] \hat{k}[\eta \hat{k} \psi]-[\eta \hat{k} \psi] \Delta \psi+\widehat{F}^{-1}\left[\gamma_{k} \psi_{k}\right]+P_{\vec{r}}
\end{array}
$$

Here dot means time-derivative, $\Delta$ - Laplace operator, $\hat{k}$ is a linear integral operator $(\hat{k}=\sqrt{-\Delta}), \widehat{F}^{-1}$ is an inverse Fourier transform, $\gamma_{k}$ is a dissipation rate (according to recent work [4] it has to be included in both equations), which corresponds to viscosity on small scales and, if needed, "artificial" damping on large scales. $P_{\vec{r}}$ is the driving term which simulates pumping on large scales (for example, due to wind). In the $k$-space supports of $\gamma_{k}$ and $P_{\vec{k}}$ are separated by the inertial interval, where the Kolmogorov-type solution can be recognized. These equations were derived as a results of Hamiltonian expansion in terms of $\hat{k} \eta$. From physical point of view $\hat{k}$-operator is close to derivative, so we expand in powers of slope of the surface. In most of experimental observations average slope of the open sea surface $\mu$ is of the order of 0.1 , so such expansion is very reasonable.

In the case of statistical description of the wave field, Hasselmann kinetic equation [5] for the distribution of the wave action $n(k, t)=\left\langle\left|a_{\vec{k}}(t)\right|^{2}\right\rangle$ is used. Here

$$
a_{\vec{k}}=\sqrt{\frac{\omega_{k}}{2 k}} \eta_{\vec{k}}+\mathrm{i} \sqrt{\frac{k}{2 \omega_{k}}} \psi_{\vec{k}},
$$

are complex normal variables. For gravity waves $\omega_{k}=\sqrt{g k}$. In this variables, if we have a linear wave with wavenumber $\vec{k}$, it will correspond to the only excited harmonics $a_{\vec{k}}$. In other words, representation in terms of these normal variables means representation in terms of elementary excitations in the system (linear waves).

\section{NUMERICAL SIMULATION.}

We simulated primordial dynamical equations (1) in a periodic spatial domain $2 \pi \times 2 \pi$. Main part of the simulations was performed on a grid consisting of $1024 \times 1024$ knots. Also we performed long time simulation on the grid

Numerical Analysis and Applied Mathematics ICNAAM 2011

AIP Conf. Proc. 1389, 693-696 (2011); doi: 10.1063/1.3636825

(C) 2011 American Institute of Physics 978-0-7354-0956-9/\$30.00 
$256 \times 256$. The used numerical code was verified in $[6,7,3,8,9,10]$. Gravity acceleration was $g=1$. Pseudo-viscous damping coefficient had the following form

$$
\gamma_{k}=\left\{\begin{array}{l}
0, k \leq k_{d} \\
-\gamma_{0}\left(k-k_{d}\right)^{2}, k>k_{d}
\end{array}\right.
$$

where $k_{d}=256$ and $\gamma_{0,1024}=2.7 \times 10^{4}$ for the grid $1024 \times 1024$ and $k_{d}=64$ and $\gamma_{0,256}=2.4 \times 10^{2}$ for the smaller grid $256 \times 256$. Pumping was an isotropic driving force narrow in wavenumbers space with random phase:

$$
P_{\vec{k}}=f_{k} \mathrm{e}^{\mathrm{i} R_{\vec{k}}(t)}, f_{k}=\left\{\begin{array}{l}
4 F_{0} \frac{\left(k-k_{p 1}\right)\left(k_{p 2}-k\right)}{\left(k_{p 2}-k_{p 1}\right)^{2}}, \\
0-\text { if } k<k_{p 1} \text { or } k>k_{p 2}
\end{array}\right.
$$

here $k_{p 1}=28, k_{p 2}=32$ and $F_{0}=1.5 \times 10^{-5} ; R_{\vec{k}}(t)$ was uniformly distributed random number in the interval $(0,2 \pi]$ for each $\vec{k}$ and $t$. Initial condition was low amplitude noise in all harmonics. Time steps were $\Delta t_{1024}=6.7 \times 10^{-4}$ and $\Delta t_{256}=5.0 \times 10^{-3}$. We used Fourier series in the following form:

$$
\eta_{\vec{k}}=\widehat{F}\left[\eta_{\vec{r}}\right]=\frac{1}{(2 \pi)^{2}} \int_{0}^{2 \pi} \int_{0}^{2 \pi} \eta_{\vec{r}} \mathrm{e}^{\mathrm{i} \vec{k} \vec{r}} \mathrm{~d}^{2} r, \quad \eta_{\vec{r}}=\widehat{F}^{-1}\left[\eta_{\vec{r}}\right]=\sum_{-N_{x} / 2}^{N_{x} / 2} \sum_{-N_{y} / 2}^{N_{y} / 2} \eta_{\vec{k}} \mathrm{e}^{-\mathrm{i} \vec{k} \vec{r}}
$$

here $N_{x}, N_{y}$ - are number of Fourier modes in $x$ and $y$ directions.

As a results of simulation we observed [1,11] formation of both direct and inverse cascades (Fig. 1, solid line). Average steepness was equal to $\sqrt{\left\langle|\nabla \eta|^{2}\right\rangle}=0.14$. What is important, development of inverse cascade spectrum was arrested by discreteness of wavenumbers grid in agreement with $[6,8,12,13]$. After that large scale condensate started to form. As one can see, value of wave action $\left|a_{k}\right|^{2}$ at the condensate region is more than order of magnitude larger than for closest harmonic of inverse cascade. Dynamics of large scales became extremely slow after this point. We managed to achieve downshift of condensate peak for one step of wavenumbers grid during long time simulation on a small grid $256 \times 256$ (Fig. 1, line with long dashes). As one can see we observed elongation of inverse cascade interval without significant change of the slope. Unfortunately, inertial interval for inverse cascade is too short to exclude possible influence of pumping and condensate.

In the case of linear waves harmonics $a_{\vec{k}}$ will have just rotation of phase with constant amplitude:

$$
a_{\vec{k}}(t)=A_{\vec{k}} \exp (-\mathrm{i} \omega t+\mathrm{i} \vec{k} \vec{r})
$$

with the dispersion relation $\omega(\vec{k})=\sqrt{g k}$.

In the case of weak nonlinearity this rotation is the fastest process, but amplitude $A_{\vec{k}}$ will be already slow function on time due to nonlinear interaction of waves. Let us investigate how the dispersion relation is influenced by the nonlinear interactions in the system. For this we record $a_{\vec{k}}(t)$ for different values of $\vec{k}$ and then calculate a Fourier transform on time, which leads to the function $a_{\vec{k}}(\omega)$. In our numerical experiment we were recording every other harmonics on the $k_{x}$-axis, from $\vec{k}=(4 ; 0)$ till $\vec{k}=(28 ; 0)$ and every tenth harmonic from $\vec{k}=(30 ; 0)$ till $\vec{k}=(250 ; 0)$ and calculated a Fourier transform. The resulting surface $\left|a_{\vec{k}}(\omega)\right|$ is represented in Figure 2. The observed sidebands are due to nonlinear interaction of waves with condensate. Although the nonlinear process supposed to be weak, the fact, that condensate is more than an order of magnitude larger in amplitude makes them essential. Here are corresponding nonlinear terms:

$$
a_{\vec{k}_{0}} a_{\vec{k}_{c}}=A_{\vec{k}} A_{\vec{k}_{c}} \exp \left[-\mathrm{i}\left(\omega\left(k_{0}\right)+\omega\left(k_{c}\right)+\mathrm{i}\left(\vec{k}_{0}+\vec{k}_{c}\right) \vec{r}\right)\right], \quad a_{\vec{k}_{0}} a_{\vec{k}_{c}}^{*}=A_{\vec{k}} A_{\vec{k}_{c}}^{*} \exp \left[-\mathrm{i}\left(\omega\left(k_{0}\right)-\omega\left(k_{c}\right)+\mathrm{i}\left(\vec{k}_{0}-\vec{k}_{c}\right) \vec{r}\right)\right],
$$

Here $\vec{k}_{c}$ - is the wavenumber of condensate and $\vec{k}_{0}$ wavenumber of some wave. The first term corresponds to the upper sideband, second - to the lower sideband. If we consider spectrum in the vicinity of condensate peak, one can see that sidebands are of close amplitudes with respect to central peak, corresponding to the $\omega(k)=\sqrt{g k}$ dispersion relation. Which means that at least for inverse cascade region distortion of the dispersion relation have to be taken into account. For the direct cascade region, which is far from the condensate scale, influence on the dispersion is almost negligible. Currently, the inverse cascade slope, observed in numerical simulations, is different from theoretical predictions of the wave turbulence theory. This result can be one of the building blocks for the theory of inverse cascade in the presence of condensate. 


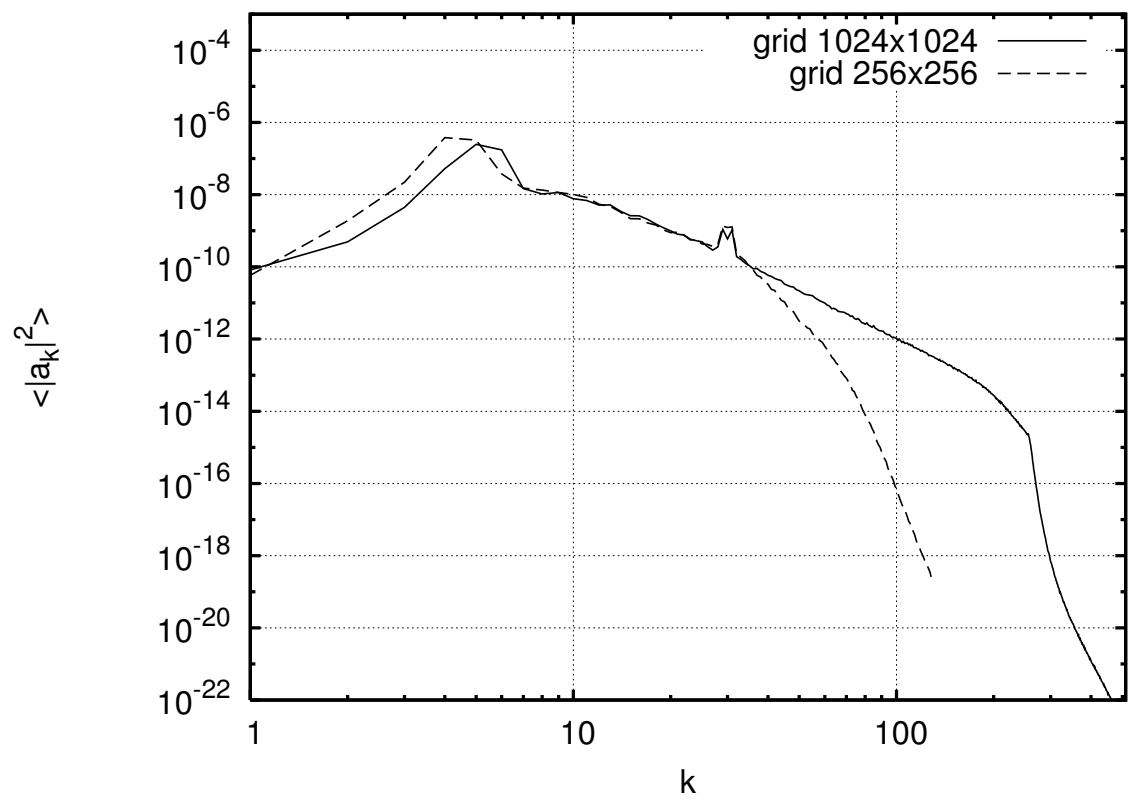

FIGURE 1. Spectra $\left\langle\left|a_{k}\right|^{2}\right\rangle$. With condensate on the $1024 \times 1024$ grid (solid); on the $256 \times 256$ grid with more developed condensate (long dashes).

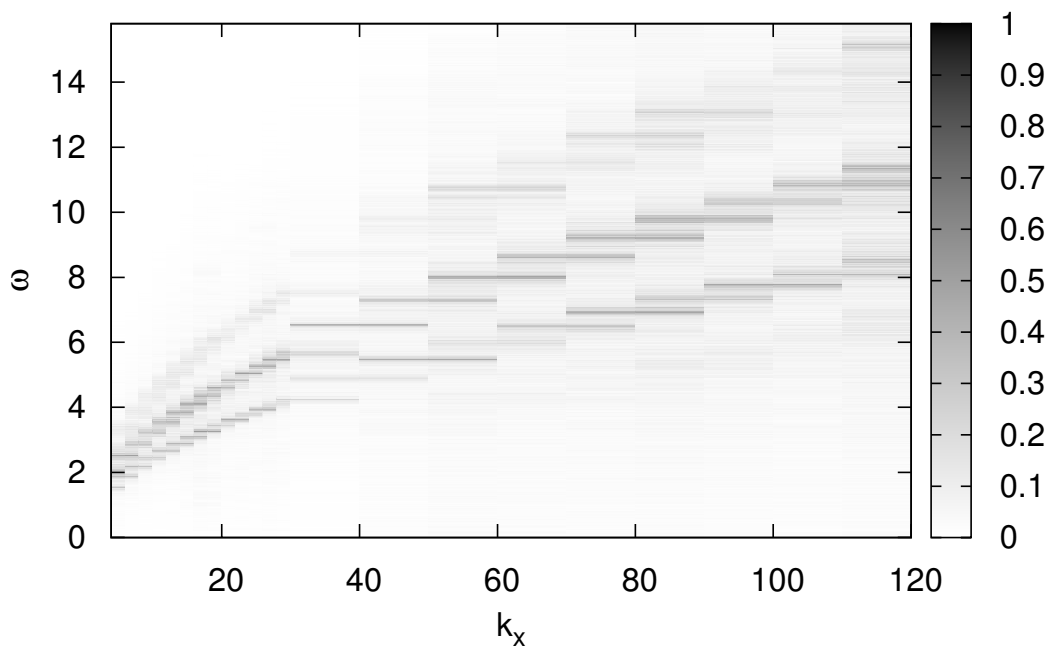

FIGURE 2. Surface $\left|a_{\vec{k}}(\omega)\right|$. Sidebands, corresponding to the interaction with condensate, are clearly visible.

\section{ACKNOWLEDGMENTS}

The author would like to thank Yu. V. Lvov and G. E. Falkovich for enlightening discussions. 
This work was partially supported by Federal Targeted Program of the Russian Federation "S\&S PPIR", the Program "Fundamental problems of nonlinear dynamics" from the RAS Presidium and "Leading Scientific Schools of Russia" grant NSh-6885.2010.2.

The author would also like to thank the creators of the free open-source fast Fourier transform library FFTW [14] for this fast, cross platform, and reliable software.

\section{REFERENCES}

1. A. O. Korotkevich, Phys. Rev. Lett. 101, 074504 (2008).

2. V. E. Zakharov, V. S. Lvov, and G. Falkovich, Kolmogorov Spectra of Turbulence I, Springer-Verlag, Berlin, 1992.

3. A. I. Dyachenko, A. O. Korotkevich, and V. E. Zakharov, Phys. Rev. Lett. 92, 134501 (2004).

4. F. Dias, A. I.Dyachenko, and V. E. Zakharov, Phys. Lett. A 372, 1297-1302 (2008).

5. K. Hasselmann, J. Fluid Mech. 12, 481-500 (1962).

6. A. I. Dyachenko, A. O. Korotkevich, and V. E. Zakharov, JETP Lett. 77, 477-481 (2003).

7. A. I. Dyachenko, A. O. Korotkevich, and V. E. Zakharov, JETP Lett. 77, 546-550 (2003).

8. V. E. Zakharov, A. O. Korotkevich, A. Pushkarev, and A. I. Dyachenko, JETP Lett. 82, 487-491 (2005).

9. V. E. Zakharov, A. O. Korotkevich, A. Pushkarev, and D. Resio, Phys. Rev. Lett. 99, 164501 (2007).

10. A. O. Korotkevich, A. Pushkarev, D. Resio, and V. E. Zakharov, Eur. J. Mech. B/Fluids 27, 361-387 (2008).

11. A. O. Korotkevich, Math. Comput. Simul., in press (2010).

12. S. V. Nazarenko, J. Stat. Mech. L02002 (2006).

13. Y. Lvov, S. V. Nazarenko, and B. Pokorni, Physica D 99, 24-35 (2006).

14. M. Frigo, and S. G. Johnson, Proc. IEEE 93, 216-231 (2005), URL http: / / fftw .org. 